\title{
Enfermedad cerebrovascular en pediatría. Experiencia de un servicio de urgencias
}

\author{
Malka Huici-Sánchez, Laura Escuredo-Argullós, Victoria Trenchs-Sáinz de la Maza, Carles Luaces-Cubells
}

Servicio de Urgencias. Hospital Sant Joan de Déu. Esplugues de Llobregat, Barcelona, España.

Correspondencia: Dra. Malka Huici Sánchez. Servicio de Urgencias. Hospital Sant Joan de Déu. Pg. Sant Joan de Déu, 2 E-08950 Esplugues de Llobregat (Barcelona)

+34932033959

E-mail:

mhuici@hsjdbcn.org

Los resultados de este estudio $s$ han presentado previamente de forma parcial como comunicación oral en el $62 .^{\circ}$ Congreso de la Asociación Española de Pediatría (Sevilla, 2013) y como póster en la 18. e Reunión de la Sociedad Española de Urgencias Pediátricas (Granada, 2013)

Aceptado tras revisión externa: 13.05.14

Cómo citar este artículo: Huici-Sánchez M, Escuredo-Argullós L, Trenchs-Sáinz de la Maza V, LuacesCubells C. Enfermedad cerebrovascular en pediatría. Experiencia de un servicio de urgencias. Rev Neurol 2014; 59: 106-10.

(c) 2014 Revista de Neurología

Objetivos. Describir las características de la enfermedad cerebrovascular (ECV) en pacientes atendidos en un servicio de urgencias pediátrico y detectar diferencias clínicas según sea isquémica o hemorrágica.

Pacientes y métodos. Estudio retrospectivo, analítico observacional, realizado en el servicio de urgencias pediátrico de un hospital de tercer nivel. Se incluyen pacientes entre 1 mes y 18 años de edad atendidos en el servicio durante 10 años (enero de 2001 a diciembre de 2011) con diagnóstico final de ECV. Se excluyen las hemorragias por traumatismos o secundarias a tumores, las trombosis de senos venosos y los pacientes no atendidos en el servicio.

Resultados. Se incluyen 61 pacientes, $39(63,9 \%)$ de sexo masculino, con una mediana de edad de 4,6 años (rango: 1,3 meses-17,5 años). De ellos, 26 (42,6\%) presentan ECV isquémica y $35(57,4 \%)$ ECV hemorrágica. La cefalea $(n=20$; $57,1 \% ; p=0,008)$ y los vómitos ( $n=25 ; 71,4 \% ; p=0,001)$ son más frecuentes en la ECV hemorrágica, y la hemiparesia ( $n=17 ; 65,4 \% ; p<0,001)$ y la parálisis facial $(n=7 ; 26,9 \% ; p=0,001)$, en la ECV isquémica. Las principales causas de la ECV hemorrágica son las malformaciones arteriovenosas $(n=17 ; 38,6 \%)$, y las de la ECV isquémica, las arteriopatías $(n=6$; 42,3\%). Al alta, 27 (44,3\%) presentaban diferentes grados de discapacidad y 6 (9,8\%) fallecieron.

Conclusiones. La ECV es una entidad poco frecuente, aunque presenta una elevada morbimortalidad. Se observa un ligero predominio de la ECV hemorrágica y se comprueba que la ECV hemorrágica se presenta más con signos de hipertensión intracraneal, y la isquémica, con focalidad neurológica.

Palabras clave. Cerebrovascular. Hemorrágico. Infancia. Isquémico. Pediátrico. Urgencias.

\section{Introducción}

La enfermedad cerebrovascular (ECV) en la infancia es una entidad infrecuente (2-13 por $100.000 \mathrm{ni}-$ ños y año en los países desarrollados) [1-3]. Se conoce poco acerca de los factores que intervienen en su etiopatogenia, evolución y pronóstico, y no se dispone de guías de actuación basadas en la evidencia [4]. A diferencia de los adultos, en los niños, la clínica de la ECV es más heterogénea e inespecífica [5-7]. Esto dificulta su diagnóstico temprano y retrasa la implantación de un tratamiento adecuado, lo que repercute en su pronóstico. Por ello, pese a su baja incidencia, representa una causa importante de discapacidad a largo plazo y es una de las 10 primeras causas de mortalidad en niños $[1,4]$, siendo tan común como los tumores cerebrales infantiles [8].

En cuanto a la etiopatogenia de la ECV en la edad pediátrica, se postula que la concurrencia de múltiples factores facilita su presentación. La presencia de factores predisponentes, como malformaciones vasculares, arteriopatías, enfermedades cardíacas o hematológicas, junto con diversos desencadenantes, como infecciones víricas, podría conducir al desarrollo de una ECV en este subgrupo de población $[4,5,9]$.
El estudio pretende describir las características principales de la ECV en pacientes atendidos en un servicio de urgencias pediátrico (SUP), e intentar detectar diferencias en su forma de presentación clínica y evolución, según sea isquémica o hemorrágica.

\section{Pacientes y métodos}

Es un estudio retrospectivo, analítico observacional, realizado en el SUP de un hospital materno infantil de tercer nivel, centro de referencia de un área de 1.800.000 habitantes, y que atiende una media de 280 visitas diarias.

Se define ECV isquémica como el déficit neurológico focal agudo de duración superior a 24 horas causado por isquemia cerebral con evidencia de infarto cerebral en neuroimagen -tomografía axial computarizada (TAC) o resonancia magnética (RM) cerebral-, correspondiente a un determinado territorio arterial que se correlaciona con la presentación clínica. Se define ECV hemorrágica como el déficit neurológico agudo de duración superior a 24 horas, con neuroimagen que objetiva hemorragia intracraneal no asociada a infarto isquémico. 
Tabla I. Antecedentes y clínica de los pacientes con enfermedad cerebrovascular, datos globales y diferencias según tipo (hemorrágica o isquémica).

\begin{tabular}{|c|c|c|c|c|c|c|c|c|}
\hline & & & otal $(n=61)$ & & emorrágica $(n=35)$ & & squémica $(n=26)$ & \\
\hline & & $n$ & \% (IC 95\%) & $n$ & \% (IC 95\%) & $n$ & $\%$ (IC 95\%) & \\
\hline & No (sano) & 40 & $65,6(53,0-76,3)$ & 22 & $62,9(46,3-76,8)$ & 18 & $69,2(50,0-83,5)$ & 0,604 \\
\hline & ECV previa/MV cerebral & 5 & $8,2(3,6-17,8)$ & 5 & $14,3(4,8-30,3)$ & 0 & - & \\
\hline & Varicela en el último año & 4 & $6,6(2,6-15,7)$ & 1 & $2,9(0,7-14,9)$ & 3 & $11,5(4,0-29,0)$ & \\
\hline Antecedentes & Migraña & 2 & $3,3(0,9-11,2)$ & 1 & $2,9(0,7-14,9)$ & 1 & $3,8(0,7-18,9)$ & \\
\hline patologicos & Historia familiar de ECV & 1 & $1,6(0,2-8,7)$ & 1 & $2,9(0,7-14,9)$ & 0 & - & \\
\hline & Drepanocitosis & 1 & $1,6(0,2-8,7)$ & 1 & $2,9(0,7-14,9)$ & 0 & - & \\
\hline & Cardiopatía & 1 & $1,6(0,2-8,7)$ & 0 & - & 1 & $3,8(0,7-18,9)$ & \\
\hline & Otros $^{a}$ & 7 & $11,5(5,7-21,8)$ & 4 & $11,4(3,2-26,7)$ & 3 & $11,5(4,0-29)$ & \\
\hline & Vómitos & 32 & $52,5(40,2-64,5)$ & 25 & $71,4(53,7-85,4)$ & 7 & $26,9(13,7-46,1)$ & 0,001 \\
\hline & Cefalea & 26 & $42,6(31,0-55,1)$ & 20 & $57,1(39,4-73,7)$ & 6 & $23,1(11,0-42,1)$ & 0,008 \\
\hline & Convulsiones & 24 & $39,3(28,0-51,9)$ & 15 & $42,9(26,3-60,6)$ & 9 & $34,6(19,4-53,8)$ & 0,515 \\
\hline & Hemiparesia & 19 & $30,6(19,6-43,7)$ & 2 & $5,7(0,6-19,2)$ & 17 & $65,4(46,2-80,6)$ & $<0,001$ \\
\hline & Alteración de la conciencia & 15 & $24,6(15,5-36,7)$ & 12 & $34,3(19,1-52,2)$ & 3 & $11,5(4,0-29,0)$ & 0,041 \\
\hline & Fiebre & 8 & $13,1(6,8-23,8)$ & 6 & $17,1(6,6-33,6)$ & 2 & $7,6(2,1-24,1)$ & 0,448 \\
\hline & Parálisis facial & 7 & $11,5(5,7-21,8)$ & 0 & - & 7 & $26,9(13,7-46,1)$ & 0,001 \\
\hline & Alteración del lenguaje & 5 & $8,2(3,6-17,8)$ & 3 & $8,6(1,8-23,1)$ & 2 & $7,6(2,1-24,1)$ & 1 \\
\hline & Alteración visual & 4 & $6,6(2,6-15,7)$ & 1 & $2,9(0,7-14,9)$ & 3 & $11,5(4,0-29,0)$ & 0,303 \\
\hline & Ataxia/dismetrías & 4 & $6,6(2,6-15,7)$ & 2 & $5,7(0,6-19,2)$ & 2 & $7,6(2,1-24,1)$ & 1 \\
\hline & $\geq 14$ & 48 & $78,7(66,9-87,1)$ & 24 & $68,6(50,7-83,1)$ & 24 & $92,3(75,9-97,9)$ & 0,073 \\
\hline $\begin{array}{l}\text { Escala de } \\
\text { Glasgow }\end{array}$ & $9-13$ & 4 & $6,6(2,6-15,7)$ & 3 & $8,6(1,8-23,1)$ & 1 & $3,8(0,7-18,9)$ & \\
\hline & $\leq 8$ & 9 & $14,8(8,0-25,7)$ & 8 & $22,9(10,4-40,1)$ & 1 & $3,8(0,7-18,9)$ & \\
\hline
\end{tabular}

ECV: enfermedad cerebrovascular; IC 95\%: intervalo de confianza al 95\%; MV: malformación vascular. a En ECV hemorrágica: hemofilia A, anemia hemolítica autoinmune, síndrome polimalformativo y prematuridad, 33 semanas de gestación; en ECV isquémica: síndrome de Down, progeria de Hutchinson Gilford y hemangiomatosis facial.

Se incluyen los pacientes entre 1 mes y 18 años de edad atendidos en el SUP durante 10 años (enero de 2001 a diciembre de 2011) y con diagnóstico final de ECV. Se excluyen las hemorragias parenquimatosas o subaracnoideas por traumatismos o secundarias a sangrado tumoral, las trombosis de senos venosos y la ECV de pacientes que no fueron atendidos en el servicio de urgencias.
Los datos de los pacientes se extraen de registros informatizados del SUP, notas de ingreso y evolución, e informes radiológicos. Se recopilan datos demográficos, antecedentes personales, clínica, pruebas de neuroimagen y conducta inicial en el SUP, pruebas complementarias realizadas para completar estudio, manejo médico e intervenciones quirúrgicas realizadas y secuelas (grado de discapaci- 
Tabla II. Intervenciones y secuelas al alta en los pacientes con enfermedad cerebrovascular, datos según tipo (hemorrágica o isquémica).

\begin{tabular}{|c|c|c|c|c|c|}
\hline & & \multicolumn{2}{|c|}{ ECV hemorrágica $(n=35)$} & \multicolumn{2}{|c|}{ ECV isquémica $(n=27)$} \\
\hline & & $n$ & \% (IC 95\%) & $n$ & \% (IC 95\%) \\
\hline \multirow{5}{*}{$\begin{array}{l}\text { Actuación } \\
\text { en SUP }\end{array}$} & TAC craneal & 29 & $82,9(67,3-92,0)$ & 19 & $73,1(53,9-86,3)$ \\
\hline & RM cerebral & 1 & $2,9(0,5-14,5)$ & 3 & $11,5(4,0-29,0)$ \\
\hline & Intubación & 10 & $28,6(16,3-43,0)$ & 3 & $11,5(4,0-29,0)$ \\
\hline & Medidas anti-HTIC & 10 & $28,6(16,3-43,0)$ & 3 & $11,5(4,0-29,0)$ \\
\hline & Traslado a la UCIP & 19 & $54,3(38,2-69,5)$ & 13 & $50(32,7-67,9)$ \\
\hline \multirow{6}{*}{$\begin{array}{l}\text { Tratamiento } \\
\text { médico }^{\mathrm{a}}\end{array}$} & Anticoagulantes & 0 & - & 8 & $30,8(16,5-50,0)$ \\
\hline & Ácido acetilsalicílico & 0 & - & 15 & $57,7(38,9-74,5)$ \\
\hline & Antiepilépticos & 23 & $65,7(49,2-79,2)$ & 10 & $38,5(22,4-57,4)$ \\
\hline & Corticoides & 20 & $57,1(39,4-73,7)$ & 16 & $61,5(42,5-77,6)$ \\
\hline & Antihipertensivos & 3 & $8,6(1,8-23,1)$ & 2 & $7,6(2,1-24,1)$ \\
\hline & Trombolíticos & 0 & - & 0 & - \\
\hline \multirow{4}{*}{$\begin{array}{l}\text { Tratamiento } \\
\text { quirúrgico }\end{array}$} & Resección de MV & 7 & $20(10,0-35,9)$ & 0 & - \\
\hline & Embolización de MV & 8 & $22,9(10,4-40,1)$ & 0 & - \\
\hline & Inserción de DVE & 11 & $31,4(18,6-48,0)$ & 0 & - \\
\hline & Craneotomía descompresiva & 2 & $5,7(0,6-19,2)$ & 0 & - \\
\hline \multirow{6}{*}{$\begin{array}{l}\text { Grado de } \\
\text { discapacidad } \\
\text { al alta }^{b}\end{array}$} & 1. Asintomático & 19 & $54,3(38,2-69,5)$ & 9 & $34,6(19,4-53,8)$ \\
\hline & $\begin{array}{l}\text { 2.Incapacidad } \\
\text { no significativa }\end{array}$ & 5 & $14,3(4,8-30,3)$ & 7 & $26,9(13,7-46,1)$ \\
\hline & 3. Incapacidad leve & 3 & $8,6(1,8-23,1)$ & 3 & $11,5(4,0-29,0)$ \\
\hline & 4. Incapacidad moderada & 2 & $5,7(0,6-19,2)$ & 4 & $15,4(2,2-33,5)$ \\
\hline & $\begin{array}{l}\text { 4 y } 5 \text {. Incapacidad } \\
\text { moderada-grave y grave }\end{array}$ & 3 & $8,6(1,8-23,1)$ & 0 & - \\
\hline & 6. Muerte & 3 & $8,6(1,8-23,1)$ & 3 & $11,5(4,0-29,0)$ \\
\hline
\end{tabular}

Anti-HTIC: medicación antihipertensión intracraneal, como suero salino hipertónico o manitol; DVE: derivación ventricular externa; ECV: enfermedad cerebrovascular; IC 95\%: intervalo de confianza al 95\%; MV: malformación vascular; RM: resonancia magnética; SUP: servicio de urgencias pediátrico; TAC: tomografía axial computarizada; UCIP: unidad de cuidados intensivos pediátrica. ${ }^{a}$ Algunos pacientes recibieron más de un tratamiento: ${ }^{b}$ Clasificación según la escala funcional de Rankin modificada [10]

dad al alta según la escala funcional de Rankin modificada [10]).

Los datos extraídos se almacenan y procesan en una base de datos relacional Microsoft Access específica. Se tabulan variables cuantitativas y cate- góricas, y posteriormente se analizan los datos con el programa SPSS v. 19.0.

\section{Resultados}

Se incluyen 61 casos, $35(57,4 \%)$ de ECV hemorrágica y 26 (42,6\%) de ECV isquémica. La edad mediana de los pacientes fue de 4,6 años (rango: 1,3 meses-17,5 años. De ellos, 18 (29,5\%; intervalo de confianza al 95\%, IC 95\% = 19,6-41,9\%) eran menores de 2 años, y 39 (63,9\%; IC 95\% = 51,4-74,8\%) eran de sexo masculino; no se hallaron diferencias en el sexo y edad de presentación entre ambos grupos. Veintiún $(34,4 \%)$ pacientes tenían antecedentes patológicos reseñables (Tabla I).

La mediana de tiempo desde el inicio de la clínica hasta la valoración en el SUP del centro de estudio fue de 10 horas (p25-75: 2-47 h) para las ECV hemorrágicas y de 14 horas (p25-75: 6-48 h) para las isquémicas, sin hallarse diferencias significativas. La tabla I muestra las manifestaciones clínicas que presentaban los pacientes al llegar al SUP.

En nueve pacientes no se identificó en el SUP que la clínica se debía a una ECV (cinco hemorrágicas y cuatro isquémicas), e ingresaron para estudio o tratamiento con otras orientaciones diagnósticas. Se trataba de cinco lactantes de 11 meses a 2 años con convulsiones, un niño de 3 años con impotencia funcional de una extremidad de tres días de evolución, un lactante de 51 días de vida con taquicardia sinusal mantenida en quien se descartó un proceso infeccioso intercurrente, y dos adolescentes, de 16 y 17 años, con orientación diagnóstica de meningitis linfocitaria y trastorno depresivo, respectivamente. La mediana de tiempo entre la atención en el SUP y el diagnóstico de ECV en estos pacientes fue de 16 horas (p25-75: 12-22 h).

La tabla II muestra las pruebas de neuroimagen y el manejo en el SUP, junto con el tratamiento médico y quirúrgico. Se realizaron de urgencias 48 TAC $(78,6 \%)$ (14, el 22,9\%, en otros centros previa derivación del paciente) y cuatro RM $(6,6 \%)$. En tres $(11,5 \%)$ de las 26 ECV isquémicas la TAC inicial no fue diagnóstica.

Durante el ingreso, todos los diagnósticos de ECV se confirmaron por neuroimagen: $60 \mathrm{TAC}(98,4 \%) \mathrm{y}$ 50 angio-RM (82\%). Se completó el estudio etiológico con ecocardiografía en 35 casos $(57,4 \%)$, estudio de trombofilia en 31 (50,8\%), y ecografía de carótidas en $21(34,4 \%)$. En la tabla III se muestran los territorios cerebrales afectos en las ECV isquémicas y las causas de las ECV halladas tras el estudio etiológico. 
Al alta, 28 niños (45,9\%; IC 95\% = 34-58,2\%) estaban asintomáticos, 27 (44,3\%; IC 95\% = 32,5-56,7\%) presentaban diferentes grados de discapacidad y 6 (9,8\%; IC 95\% = 4,6-19,8\%) habían fallecido.

\section{Discusión}

Este trabajo constata que, a pesar de la escasa prevalencia de la ECV en urgencias pediátricas (media de siete casos al año), supone una importante morbimortalidad en la población infantil. También se observa un ligero predominio de la ECV hemorrágica $(57,4 \%)$ respecto a la isquémica $(42,6 \%)$, coincidiendo con otras series publicadas $[3,11,12]$. En relación con la edad y el sexo, no se han encontrado diferencias significativas entre ambos tipos de ECV, y se ha observado una mediana de edad de presentación menor (4,6 años) que en la bibliografía. Esto podría deberse a que en este estudio se excluyen pacientes postraumáticos o con trombosis de senos venosos, que son más frecuentes en niños de mayor edad.

Como describen la mayoría de los estudios revisados, las malformaciones vasculares son el principal factor de riesgo para la ECV hemorrágica, y la arteriopatía estenooclusiva, para la isquémica [13]. A diferencia de otras series, existe un alto porcentaje $(65,6 \%)$ de niños previamente sanos, probablemente por los criterios de exclusión de la muestra, que excluye los casos de ECV durante la hospitalización por otras causas (meningitis, sepsis, tumores cerebrales, etc.) o en contexto quirúrgico.

Se constata que el tiempo desde el inicio de los síntomas hasta el momento de la consulta es prolongado [5], lo que dificulta la evaluación en niños de terapias de instauración precoz (primeras 3-6 h), como los trombolíticos.

A pesar de la heterogeneidad clínica, se comprueba que la ECV hemorrágica en niños se presenta más frecuentemente con signos de hipertensión intracraneal, y la isquémica, con focalidad neurológica, siendo la clínica más inespecífica en los menores de 6 años [12].

Al igual que en otros trabajos, se realizó una prueba de imagen inicial en el SUP a todos los pacientes en los que se sospecha una ECV. Sin embargo, en un 11,5\% de los casos con diagnóstico final de ECV, la inespecificidad de la clínica inicial dificultó su sospecha, por lo que no se realizó prueba de imagen inicial y se demoró el diagnostico y el tratamiento [14]. La TAC craneal inicial resultó diagnóstica en la mayoría de los casos de ECV isquémica (88,5\%), siendo los restantes confirmados por RM cerebral. Por ello, esta última podría ser la
Tabla III. Causas de la enfermedad cerebrovascular hemorrágica e isquémica.

\begin{tabular}{|c|c|c|c|c|}
\hline & & & $n$ & $\%$ (IC 95\%) \\
\hline \multirow{6}{*}{$\begin{array}{l}\text { Enfermedad } \\
\text { cerebrovascular } \\
\text { hemorrágica } \\
(n=35)\end{array}$} & \multirow{6}{*}{ Causa } & Malformación arteriovenosa & 11 & $31,4(18,6-48,0)$ \\
\hline & & Hemorragia subaracnoidea & 9 & $25,7(14,2-42,1)$ \\
\hline & & Cavernoma & 6 & $17,1(6,6-33,6)$ \\
\hline & & Coagulopatía a & 2 & $5,7(0,6-19,2)$ \\
\hline & & Síndrome moya-moya & 1 & $2,9(0,5-14,5)$ \\
\hline & & No filiada & 6 & $17,1(6,6-33,6)$ \\
\hline \multirow{10}{*}{$\begin{array}{l}\text { Enfermedad } \\
\text { cerebrovascular } \\
\text { isquémica } \\
(n=26)\end{array}$} & \multirow{3}{*}{$\begin{array}{l}\text { Circulación } \\
\text { afectada }\end{array}$} & Anterior completa & 8 & $30,8(16,5-50,0)$ \\
\hline & & Posterior completa & 7 & $26,9(13,7-46,1)$ \\
\hline & & Anterior y posterior & 11 & $42,3(25,5-61,1)$ \\
\hline & \multirow{7}{*}{$\begin{array}{l}\text { Clasificación } \\
\text { de subtipos } \\
\text { pediátricos }\end{array}$} & Arteriopatía estenooclusiva & 11 & $42,3(25,5-61,1)$ \\
\hline & & Indeterminada ${ }^{b}$ & 8 & $30,8(16,5-50,0)$ \\
\hline & & Disección arterial & 2 & $7,6(2,1-24,1)$ \\
\hline & & Cardioembolismo $^{C}$ & 2 & $7,6(2,1-24,1)$ \\
\hline & & Otras causas determinadas ${ }^{d}$ & 1 & $3,8(0,7-18,9)$ \\
\hline & & Síndrome moya-moya & 1 & $3,8(0,7-18,9)$ \\
\hline & & Causa posible $\mathrm{e}^{\mathrm{a}}$ & 1 & $3,8(0,7-18,9)$ \\
\hline
\end{tabular}

IC 95\%: intervalo de confianza al 95\%. ${ }^{a}$ Un lactante de 39 días con enfermedad hemorrágica del recién nacido tardía y un lactante de 13 meses con hemofilia $\mathrm{A} ;{ }^{\mathrm{b}}$ Todos con estudio protrombótico, angiorresonancia magnética cerebral y ecocardiograma negativos; ' Uno de los niños, de 2,8 años, diagnosticado de déficit de proteína C; d Progeria de Hutchinson Gilford; ${ }^{e}$ Inmunodeficiencia variable común (síndrome de Evans).

mejor prueba inicial para su detección, aunque la menor accesibilidad, el coste económico y la necesidad de sedación limitan su uso como técnica diagnóstica urgente.

El tratamiento de la ECV en la infancia se basa en el consenso de expertos, que está limitado por la falta de estudios realizados (con la notable excepción de los estudios centrados en la enfermedad de células falciformes, cuyo tratamiento es específico). El uso de antiepilépticos continúa siendo controvertido como profilaxis y, en relación con el uso de los corticoides, se ha demostrado en varios estudios de adultos su poca eficacia en el descenso de la presión intracraneal con aumento de la glucemia, por lo que actualmente no está recomendado [4]. En nuestra serie se observa una gran proporción de uso de antiepilépticos y de corticoides, probablemente 
por la variabilidad en el tiempo del manejo terapéutico de estos pacientes.

Casi la mitad de los supervivientes presentó algún grado de discapacidad, lo que concuerda con otros estudios $[5,14]$. La mortalidad en nuestra cohorte fue del 9,8\%, algo más baja que la notificada en otras series, con una mortalidad del $15-25 \%[5,15]$. Esto, de nuevo, podría explicarse por los criterios de selección de la muestra, que excluye patologías que conllevan un peor pronóstico. Los números absolutos insinúan una mayor mortalidad y más secuelas neurológicas graves en la ECV hemorrágica, lo que coincide con algunos estudios publicados [9].

El carácter retrospectivo de este estudio constituye su principal limitación. Por otro lado, el reducido tamaño de la muestra hace que los resultados deban interpretarse con cautela.

Como conclusión, hay que resaltar que es importante sospechar la ECV en un SUP para poder realizar un diagnóstico y tratamiento precoces que aseguren un mejor pronóstico.

Bibliografía

1. Lynch JK, Hirtz DG, DeVeber G, Nelson KB. Report of the National Institute of Neurological Disorders and Stroke workshop on perinatal and childhood stroke. Pediatrics 2002; 109: 116-23.

2. Beslow LA, Licht DJ. Predictors of outcome in childhood intracerebral hemorrhage, a prospective consecutive cohort study. Stroke 2010; 41: 313-8.

3. Fullerton HJ, Wu YW, Zhao S, Johnston SC. Risk of stroke in children: ethnic and gender disparities. Neurology 2003; 61:189-94.

4. Jordan LC, Hills AE. Challenges in the diagnosis and treatment of pediatric stroke. Neurology 2011; 7: 199-208.

5. Chriterson R, Römberg B. Childhood stroke in Sweden I: incidence, symptoms, risk factors and short-term outcome. Acta Paediatr 2010; 99: 1641-9.

6. McGlennan C, Ganesan V. Delays in investigation and management of acute arterial Ischaemic stroke in children. Dev Med Child Neurol Suppl 2008; 50: 537-40.

7. Rafay MF, Pontigon AM, Chiang J, Adams M, Jarvis DA, Silver F, et al. Delay to diagnosis in acute pediatric arterial ischemic stroke. Stroke 2009; 40: 58-64.

8. Kaatsch P, Rickert CH, Kühl J, Schüz J, Michaelis J. Populationbased epidemiologic data on brain tumors in German children. Cancer 2001; 92: 3155-64.

9. Cardo E, Pineda M, Vilaseca MA, Artuch R, Campistol J. Factores de riesgo de la enfermedad cerebrovascular en la infancia. Rev Neurol 2000; 30: 21-7.

10. Bonita R, Beaglehole R. Recovery of motor function after stroke. Stroke 1988; 12: 1497-500.

11. Wraige E, Pohl KR, Ganesan V. A proposed classification for subtypes of arterial ischaemic stroke in children. Dev Med Child Neurol Suppl 2005; 47: 252-6.

12. Warren D. Childhood hemorrhagic stroke: an important but understudied problem. J Child Neurol 2011; 26: 1174.

13. Marckay M, Wiznitzer M, Benedict S, Lee K, DeVeber G, Ganesan V. Arterial ischemic stroke risk factors: the International Pediatric Stroke Study. Ann Neurol 2011; 69: 130-40.

14. Yock-Corrales A, Mackay MT, Mosley I, Maixner W, Babl FE. Acute childhood arterial ischemic and hemorrhagic stroke in the emergency department. Ann Emerg Med 2011; 58: $156-63$.

15. Tomás-Vila M, Vázquez-Costa JF, Izquierdo-Sebastiá S, Tellez de Meneses-Lorenzo M, Dasí-Carpio MA, MulasDelgado F, et al. Enfermedad cerebrovascular en la infancia. Análisis retrospectivo de los ingresos hospitalarios en los últimos diez años en un hospital terciario de la Comunidad Valenciana. An Pediatr (Barc) 2012; 77: 75-82.

\section{Stroke in children. Experience in an emergency service}

Aims. To describe the characteristics of cerebrovascular disease (CVD) in patients treated in a paediatric emergency department and to detect clinical differences, depending on whether the condition was ischaemic or haemorrhagic.

Patients and methods. An analytical, observation-based, retrospective study was conducted in the paediatric emergency department of a tertiary care hospital. The sample included patients aged between 1 month and 18 years who were treated in the service over a period of 10 years (January 2001 to December 2011) with a final diagnosis of CVD. Haemorrhages due to traumatic injury or secondary to tumours, thrombosis of the venous sinuses and patients who were not treated in the service were all excluded.

Results. The final sample consisted of 61 patients, 39 (63.9\%) of whom were males, with a mean age of 4.6 years (range: 1.3 months-17.5 years). Of them, 26 (42.6\%) presented ischaemic CVD and 35 (57.4\%) had haemorrhagic CVD. Headache ( $n=20 ; 57.1 \% ; p=0.008)$ and vomiting $(n=25 ; 71.4 \% ; p=0.001)$ are more frequent in haemorrhagic CVD, and hemiparesis $(n=17 ; 65.4 \% ; p<0.001)$ and facial palsy $(n=7 ; 26.9 \% ; p=0.001)$ in ischaemic CVD. The main causes of haemorrhagic CVD are arteriovenous malformations $(n=17 ; 38.6 \%)$ and the ischaemic ones are triggered by arteriopathies ( $n=6 ; 42.3 \%)$. On discharge from hospital, 27 (44.3\%) presented different degrees of disability and 6 (9.8\%) died.

Conclusions. CVD is a rare entity, although it presents a high morbidity and mortality rate. Haemorrhagic CVD is seen to predominate slightly and it is observed how haemorrhagic CVD presents more often with signs of intracranial hypertension, while the ischaemic form tends to have more neurological focus.

Key words. Childhood. Emergency. Hemorrhagic. Ischemic. Pediatric. Stroke. 